\title{
Genomic Heterogeneity Contributed To The Different Survival Between Adult and Pediatric Acute Lymphoblastic Leukemia
}

\section{Yanxin Chen}

Fujian Institute of Hematology

\section{Yongzhi Zheng}

Fujian Institute of Hematology

\section{Yunda Hong}

Fujian Institute of Hematology

\section{Jingjing Wen}

Fujian Institute of Hematology

Jiazheng Li

Fujian Institute of Hematology

\section{Yan Huang}

Fujian Institute of Hematology

\section{Yi Chen}

Fujian Institute of Hematology

Jianda Hu ( $\nabla$ drjiandahu@163.com )

Fujian Institute of Hematology

\section{Research}

Keywords: acute lymphoblastic leukemia, NGS, genomic profiling, heterogeneity

Posted Date: November 9th, 2021

DOI: https://doi.org/10.21203/rs.3.rs-1035552/v1

License: (c) (1) This work is licensed under a Creative Commons Attribution 4.0 International License. Read Full License 


\section{Abstract}

Prognosis of acute lymphoblastic leukemia (ALL) in adults is inferior to children. Hence, ALL is still a challenging disease to be cured in adult population. Aberrant genetic alterations have been observed previously in $A L L$, while the patterns of differential gene alteration have not much been comprehensively determined in the adult and pediatric ALL on a genome-wide scale. This study was attempted to investigate the biological differences in genomic profiling between the adults and children with ALL, and the relationship between the genomic heterogeneity and prognosis. The results showed the similar common mutation types in two populations but the increased prevalence of genetic alterations in adult ALL. The median number of detected gene mutations was 17 (range: 1-53) per sample in adult ALL and 4.5 (range: $1-19)$ in pediatric $A L L(P<0.001)$. A significant correlation between the increased number of gene mutations and age was found $\left(R^{2}=0.5853, P<0.001\right) .122$ and 53 driver genes were identified in adult and pediatric ALL samples, respectively. The IKZF1, IDH1 and TTN mutations were significantly enriched in adult ALL. The incidence of relapse was $40.0 \%$ and $9.6 \%$ in the adult and pediatric ALL, respectively $(P=0.0003)$. The overall survival (OS) and relapse free survival (RFS) of adult ALL were poorer than pediatric ALL $(P=0.0002, P<0.001$, respectively). This genomic landscape enhanced the understanding of the biological differences of ALL between the two populations and provided a clue for novel therapeutic approaches.

\section{Introduction}

Acute lymphoblastic leukemia (ALL) is a heterogeneity aggressive hematological malignancy, which occurs more frequently in the pediatrics than the adults. Benefited from the development of treatment strategies, including the intensified chemotherapy, hematopoietic stem cell transplantation and chimeric antigen receptor-T-cell immunotherapy(1), survival of ALL was improved, particularly in pediatric patients. However, prognosis of in adult ALL cases remain unsatisfactory. Approximate $30 \%-40 \%$ adults patients with ALL experienced an relapse soon after complete remission(CR) and eventually developed refractory leukemia associated with a poor likelihood of cure, with a 5-year overall survival (OS) rate of about $50 \%$ (2). Hence, ALL is still a challenging disease to be cured in adult population.

Aberrant genetic alterations and molecular abnormalities have been revealed to be involved in the disease progression of ALL, affecting the cell differentiation, cell proliferation and drug resistance(3-6). Several biomarkers with important roles in molecular pathogenesis and clinical significance were identified to risk stratification and prognosis prediction(7), which provided potential therapeutic targets and informed the selection of specific therapies. Of note, the application of tyrosine kinase inhibitors (TKI) improved the prognosis of ALL patients with BCR-ABL1 transcripts(8). Recently, advance in next-generation sequencing (NGS) technologies, such as whole-exome sequencing (WES) and RNA sequencing (RNA-seq), have extended the recognition of genetic abnormalities in ALL, including DNA sequence abnormalities, aberrant epigenetic modifications and gene expression anomalies(9-11). However, the potential contribution of these genetic or epigenetic aberrations, especially in adult patients, needs to be further investigated to refine the route of leukemogenesis and clone evolution of ALL. 
High frequency of unfavorable genetic subtype of adult patients in part contributed to the inferior prognosis in ALL. For example. ALL patients with BCR-ABL1 are uncommon in children $(2 \%-5 \%$ of patients) but accounts for at least $25 \%$ of adults(12). In MLL-rearranged ALL, older children had higher frequency of somatic mutations and epigenetic regulators mutations than infants(13). Moreover, despite the same genetic subtype, compared with the pediatric ALL patients, the adult patients are more insensitive to the standard therapy. The differences of genetic background and treatment response in adult and children ALL indicated the distinction of leukemogenesis and pathogenesis between the two groups.

To get more insights into the genetic heterogeneity of ALL, especially in adult group, this study was attempted to investigate the biological differences in genomic profiling between the adults and children with ALL, and the possible correlation between the genomic heterogeneity and prognosis.

\section{Methods}

\section{Patients}

64 adults and 54 children patients with ALL in our institution were studied. All patients were de novo and treated with standard chemotherapy regimens. Clinical data including age, gender, hematologic laboratory values, immunologic phenotype, cytogenetic profile, Philadelphia chromosome (Ph) status, blast percentage of bone marrow and peripheral blood, achievement of complete remission (CR), overall survival (OS) and relapse free survival (RFS) were collected on all patients. Risk stratification criteria was on guidelines of the National Comprehensive Cancer Network (NCCN)(14). Philadelphia chromosome positivity $(\mathrm{Ph}+)$ defined as the existence of either BCR-ABL1 fusion gene mRNA using RT-PCR or FISH analysis, or $\mathrm{t}(9 ; 22)(\mathrm{q} 34.1 ; \mathrm{q} 11.2)$ by cytogenetic assay in the peripheral blood or bone marrow. Ph-neg patients received standard chemotherapy. $\mathrm{Ph}+$ patients received standard chemotherapy plus tyrosine kinase inhibitor (TKI). Some of patients received bridge hematopoietic stem cell transplantation (HSCT). The study was approved by the Fujian Union Hospital Institutional Review Board. Informed consent or assent was obtained from all subjects and/or their legal guardians.

\section{Exome sequencing}

Exome sequencing was performed by Kindstar (Wuhan, $\mathrm{CN}$ ). In general, bone marrow or peripheral blood samples from the de novo ALL patients were collected. Mononuclear cells were separated by Ficoll gradient according to the manufacturer's guidelines. Genomic DNA was extracted and sheared to 150-250 bp using the Covaris platform. Exome was captured using Agilent Sureselect Human All Exon V6A kit (CA, USA). Exome libraries was sequenced through Illumina high throughput sequencing system. The primary image data derived from Illumina sequencing system was transformed to the paired-end reads by the Base Calling software, and stored in a format of FASTQ, named as raw data.

\section{Whole exome sequencing analysis}


Low-quality variations (quality score $\geq 20$, Q20) were filtered. The qualified clean data, paired-end reads were aligned to the reference NCBI human genome assembly GRCh37/HG19-lite using BWA69 (version 0.5.10). Repetitive PCR fragments was deleted by Picard-tools. Read depth assessment, coverage and quality detection were performed. Single nucleotide polymorphism (SNP) and tiny oligonucleotide fragments indel/detection were detected by Genome Analysis Toolkit (GATK) software and annotated using Annovar software. Synonymous changes and SNPs that MAF determined to be higher than $5 \%$ were removed (http://www.ncbi.nlm.nih. gov/projects/SNP). Nonsynonymous changes and small indels were filtered using SIFT software (version 1.03). Variants associated with no-functional, or truncatingproteins were defined as deleterious mutations, including frameshift mutations, splice site mutations, and stop-gain mutations.

\section{RNA sequencing and analysis}

Total RNA was isolated from ALL samples of bone marrow using TRIzol. The median blast infiltration (\%) of the bone marrow were $56 \% \pm 21 \%$ in adult patients and $50 \% \pm 17 \%$ in pediatric patients. Ribosomal RNA was removed using Ribo-Zero rRNA removal beads. cDNA library was constructed using Illumina TruSeq stranded Total RNA Kit and enriched using PCR amplification. The enriched cDNA libraries were sequenced through Illumina high throughput sequencing system. Sequencing data analysis includes data preprocessing and quality control. Data qualified for quality control is performed using software such as Fusion Catcher and SOAPfuse Fusion gene detection.

\section{Driver gene identification}

The driver genes of the mutated genes were selected from the COSMIC platform (https://cancer.sanger.ac.uk/census), a web-based database for providing the information of a catalogue of genes with mutations that are causally implicated in cancer.

\section{Gene ontology and pathway enrichment analyses}

The DAVID (https://david.ncifcrf.gov/conversion.jsp), a web-based platform for gene functional annotations and biological meaning elucidation, was used to perform $\mathrm{GO}$ enrichment analysis. Pathway analysis allows to understand molecular interactions in the pathway maps. Pathway enrichment analysis was generated by the Kyoto Encyclopedia of Genes and Genomes (KEGG), which were included in the DAVID website, with $\mathrm{P}<0.05$ as the threshold value.

\section{Integration of protein-protein Interaction (PPI) Network}

String JAVA platform (http://string-db.org/), an online biomolecular protein-association network platform, was utilized to expand the DEG-encoded proteins and protein-protein interaction (PPI) network. Furtherly the PPI network was imported into Cytoscape software for constructing protein interaction link and accessing the interaction relationship of genes encoding proteins of ALL.

\section{Statistical analysis}


Associations among categorical variables or continuous parameters were determined using two-sided Fisher's exact test. Distribution differences were assessed with Wilcoxon-Mann-Whitney's test. CR required documentation of a bone marrow biopsy with cytology analysis and flow cytometry when available. OS was defined as the intervals between the date of initial diagnosis and the date of death for any cause, with censoring of last follow-up. For those patients achieving CR, relapse free survival (RFS) was calculated and defined as time from determined CR to relapse or when follow-up was terminated. RFS and OS were compared by the log-rank test and calculated by the Kaplan-Meier estimator. Analyses were performed by using Prism version 8.0 (GraphPad Software), R version 3.4.3 and SPSS software version 26.0 (SPSS, Chicago, IL, USA). Two-sided $\mathrm{P}<0.05$ was considered statistically significant.

\section{Results}

\section{Comparisons of clinical characteristics between adult-ALL and pediatric-ALL}

Among the 64 adult ALL patients, 34(53.12\%) were females and 30(46.88\%) were males, including 55(85.93\%) B-cell ALL (B-ALL) and 9(14.07\%) T-ALL. The median diagnosis age was 38.5(range, 14-76 years). As to the 54 children ALL patients, 23(42.5\%) were females and 31(57.4\%) were males, with 49(90.7\%) B-ALL and 6(9.3\%) T-ALL. The mean diagnosis age was 4.9 (range, 0.3-13.8 years). Compared with pediatric patients, the de novo adult ALL had higher abnormal karyotype (48.4\% vs. $18.5 \%, \mathrm{P}=0.002)$ and relapse rate $(40.0 \%$ vs. $9.6 \%, P=0.0003)$, and inferior tendency of CR rate ( $85.9 \%$ vs. $92.6 \%$, $\mathrm{P}=0.0634)$. There were no differences in gender, initial white blood cell (WBC) counts, hemoglobin level, platelets level, immunophenotype, LDH level. Clinical characteristics of adult-ALL and pediatric-ALL patients at diagnosis and efficacy of treatment are described in Table 1.

Analysis of transcriptome sequencing data identified transcripts abnormal derived from 66 gene rearrangements in 38 (59.3\%) adult ALL samples and 20 gene rearrangements in 15 (27.7\%) pediatric ALL samples. The most frequent gene rearrangements were BCR-ABL1 fusions (18 samples), fusions of PAX5-ZCCHC7 (6 samples) in adult ALL. (Figure1A)

\section{Comparison of mutation frequencies between adult and pediatric cases}

To explore the possible genetic differences between adults and children, we compared the total number of non-silent mutations in the tested cohort and found that it was significantly higher in adults than in children. All the adult-ALL and pediatric-ALL patients harbored somatic mutations. The sequencing revealed 1013 high-confidence somatic single-nucleotide variants (SNVs), 48 stop gain and 23 frameshift insertion in 1022 genes in the 39 adult ALL samples. While in the 54 pediatric ALL samples, 232 SNVs, 12 stop gain and 10 frameshift insertion in 284 genes were identified. The results showed the similar common mutation types in adult and pediatric ALL. (Supplement Table1, Figure 1B, 1C) 
To investigate the potential genetic difference between the patients with adult ALL and pediatric ALL, we compared the mutation frequencies of samples from the two group. The results showed that the median number of detected gene mutations was 17 (range: 1-53) per sample in adult ALL and 4.5 (range: 1-19) in pediatric $A L L(P<0.001)$, indicating the increased prevalence of genetic alterations in adult ALL (Figure 1D). Moreover, we divided the age into children ( $<14 y)$, adolescent and young adult (14-35y) and adult (>35y) and compared the number of detected gene mutations. Results showed that elevation rate of genetic abnormal in the adolescent and young adult (14-35y) and adult (>35y) compared with children $(<14 y)(P<0.001$, Figure 1E). While no significant difference was observed in the young adult (14$35 y)$ and adult $(>35 y)(P>0.05)$, Additionally, a significant correlation between the increased of number of gene mutations and age was found $\left(R^{2}=0.5853, P<0.001\right.$, Figure $\left.1 F\right)$.

\section{Comparison of genetic features between adult and pediatric cases}

The most frequently mutated genes were NRAS in 9 samples (14.06\%), TTN in 6 samples $(9.38 \%)$, NOTCH1 in 6 samples (9.38\%) and IKZF1 in 6 samples (9.38\%) in adult ALL. In pediatric ALL, the most frequently mutated genes were KRAS in 15 samples (27.7\%), NOTCH1 in 13 samples (24.1\%), NRAS in 9 samples (16.7\%) and CREBBP in 6 samples (11.1\%) (Figure 2A). The IKZF1, IDH1 and TTN mutations were significantly enriched in adult ALL. While the KRAS, ARID1A and CREBBP mutations were significantly enriched in pediatric ALL (Figure 2B). Moreover, TP53 mutation was found in 4/64(6.25\%) adult and 2/54(4\%) pediatric ALL samples.

122 and 53 driver genes were identified in adult and pediatric ALL samples, respectively (Table2). To understand difference of the functional changes of adult-ALL and pediatric ALL, the above driver genes were mapped on the DAVID database for GO analysis. The primary GO terms of biological process (BP) enriched in the drivers genes of adult ALL were positive regulation of transcription from RNA polymerase II promoter, positive regulation of transcription from RNA polymerase II promoter, positive regulation of transcription from RNA polymerase II promoter, signal transduction, positive regulation of transcription, DNA-templated $(P<0.05)$ (Figure $3 A$ ). As to pediatric $A L L$, the enriched $G 0$ terms of $B P$ in the driver genes were regulation of double-strand break repair, $\mathrm{N}$-terminal peptidyl-lysine acetylation, MAPK cascade and regulation of myeloid cell differentiation $(P<0.05)$ (Figure $3 C$ ).

Using integrated genomic analysis, we identified 4 functional pathways recurrently mutated in adult ALL: Pathways in cancer, HTLV-I infection, PI3K-Akt signaling pathway, and Proteoglycans in cancer $(\mathrm{P}<0.05)$ (Figure 3B), 5 in pediatric ALL: Transcriptional regulation, PI3K-AKT-mTOR signaling, JAK-STAT signaling, NOTCH1 signaling, FoxO signaling pathway $(P<0.05)$ (Figure 3D).

\section{Comparison of difference of hub genes between adult and pediatric cases}

String database and Cytoscape software were employed to identify and visualize the PPI network derived from those above driver genes of adult and pediatric ALL. For adult ALL, a total of 122 out of the 122 
driver genes were inserted in the PPI network, which comprised 122 nodes and 1178 edges (molecular interaction) with PPI enrichment $\mathrm{P}$ value of $<1.0 \mathrm{e}-16$, indicating a reliable driven genes PPI network. By Cytoscape MCODE analysis, 5 significant modules were screened from the PPI network (Figure 4A). Of these, module 1 included 58 nodes and 1526 edges, module 2 included 25 nodes and 266 edges. Using cytoHubba analysis, among the 101 nodes, TP53, KRAS, CDKN2A, NF1, NOTCH1, STAT3, SMARCA4, CREBBP, NRAS, PTPN11, the ten central node genes with degree $>10$, were considered as hub driver genes in the PPI network (Figure 4B).

As to pediatric ALL, a total of 53 out of the 53 driver genes were inserted in the PPI network, which comprised 53 nodes and 281 edges (molecular interaction) with PPI enrichment $P$ value of $<1.0 \mathrm{e}-16$, indicating a reliable driven genes PPI network. By Cytoscape MCODE analysis, 2 significant modules were screened from the PPI network (Figure 4C). Of these, module 1 included 14 nodes and 55 edges, and module 2 included 13 nodes and 33 edges. Using cytoHubba analysis, among the 53 nodes, MYC, EZH2, CDKN2A, CREBBP, SMARCA4, ATRX, NF1, EP300, NOTCH1, BRCA1, the ten central node genes with degree $>10$, were considered as hub driver genes in the PPI network (Figure 4D).

\section{Associations of genetic features with clinical outcomes in adult and pediatric cases}

The incidence of relapse was $40.0 \%$ and $9.6 \%$ in the adult and pediatric ALL, respectively $(P=0.0003)$. The overall survival (OS) and relapse free survival (RFS) of adult ALL were poorer than pediatric ALL $(P=0.0002, P<0.001$, respectively, Figure $5 A, B)$, indicating an unfavorable prognosis.

We furtherly investigated the effect of gene mutation on the survival of ALL in adult and pediatric case. There are three genes overlapping between the high frequency (HF) mutated genes and the hub driver genes of adult ALL and pediatric ALL, including NOTCH1, CREBBP and SMARCA4. (Figure 6A)

The clinical significance of these above overlapped genes was examined using the survival analysis. The results showed no survival difference between the mutated cases and wild type case of the three genes in adult ALL and pediatric ALL (Figure 6B-D).

\section{Discussions}

Aberrant genetic alterations and molecular abnormalities have been identified to be associated with the disease progression of ALL. In this study, we analyzed the genomic data and clinical characteristics, attempted to identify the significance of genetic alterations for prognostication in adult ALL and pediatric ALL.

The adult patients had more gene mutations than the pediatric patients, which is in accordance with previous studies $(15,16)$. The median number of detected gene mutations was 19 (range: $1-53$ ) per sample in adult ALL and 4.5 (range: 1-19) in pediatric $\operatorname{ALL}(P<0.001)$, in which the SNV was the most common mutation types of the genomic profiling, that were in accordance with which reported in 
previous studies(17-19). In adult ALL, the most frequently mutated genes were NOTCH1(15.38\%), TTN $(12.8 \%)$ and IKZF1(10.2\%) respectively. In pediatric ALL, the most frequently mutated genes were KRAS (27.7\%), NOTCH1 $24.1 \%)$, NRAS (16.7\%) and CREBBP (11.1\%) respectively. The frequency of mutation in genomic profiling was comparable to the data previously reported $(20,21)$.

Using the bioinformatic tools, the different hub driver genes were screened out. Adult ALL patients had more enrichment for alterations of epigenetics and tumor metabolism, such as IDH1, DNMT3A, which may explain in part the disparity in the different responses to treatment of the two populations. IDH1 mutations are most often discovered in acute myeloid leukemia and myelodysplastic syndrome(22). Besides, IDH1 mutations are also detected in a small proportion of adult T-cell acute lymphoblastic leukemia (T-ALL)(23). It's reported that mutations of IDH1 contributed to the pathogenesis of T-cell malignancies in mice and probably increase the metabolic profile of malignant T cells (24). DNA methyltransferase DNMT3A is important for formation of mammalian DNA methylation during development. Mutations of DNMT3A are frequently found in AML, which associated with poor prognosis. DNMT3A mutations as key predictors of inferior OS in AML patients $<60$ years, and particularly in those with intermediate-risk cytogenetics(25). Alterations of DNMT3A in lymphoid malignancies are less frequently, and reports to date are confined to T-lineage disease(26). Jonathan et al reported that mutations of DNMT3A were strongly correlated with increased age and inferior prognosis in adult T-cell acute lymphoblastic leukemia(27).

It's reported that NOTCH1 mutations was presented in the $65 \%$ of patients with T-ALL, that induced the continuous activation of NOTCH1-MYC pathway and furtherly contributed to the promotion of the proliferation of the leukemic cells(28). IKZF1 mutations accounted for $15 \%$ of the patients with B-ALL and $70 \%$ of the patients with $\mathrm{Ph}$ positive and $\mathrm{Ph}$ like ALL, with inferior survival. TTN gene variant was related to a low cardiorespiratory fitness level in pediatric ALL(29). However, the reports about TTN mutations in the adult-ALL was poor. Notably, in our study, TTN mutations occurred in the patients with adult-ALL, but not detected in patients with pediatric ALL. Recently, largescale of sequencing studies have revealed high prevalence of Ras mutations in the pediatric malignancies. Ras mutations resulted in the continuous activation of Ras pathway, mediated the resistance to chemotherapy and the disease progression $(30,31)$. In our cohort, we also observed the high frequency mutation of Ras gene in the pediatric ALL. CREBBP gene was functions with the histone modification as a transcriptional co-activator. CREBBP mutations were common in hematological malignancies and with the clinical relevance and prognostic value in the relapse of pediatric $\operatorname{ALL}(32,33)$. The future studies will enlarge the cohorts and prolonger the follow up time to explore the effect of the above mutated genes on the efficacy and survival of ALL.

In our cohort, the hub driver genes did not display the survival value, may be due to the limit of the sample size, the follow up time, the improvement of the treatment. The further study will be continuous to enlarge the cohort and expand the observe time.

Our study revealed a significant correlation between the increased of number of gene mutations and age $\left(R^{2}=0.3096, P<0.001\right)$. This suggests that the occurrence of ALL in elderly patients may be related to the 
progressive decline of hematopoietic function and mutation accumulation with age; while children's patients may have genetic predisposing factors, which can occur without or accumulating fewer somatic gene mutations. Research results show that there is age-related clonal hematopoiesis in the elderly, and it is related to the occurrence of $\operatorname{ALL}(34)$. There are significant differences in the genetic background and the law of disease in patients of different ages. Discover and identify the patient's genetic predisposing factors for better treatment options.

\section{Conclusions}

The study in genomic profiling across the age spectrum elucidated the genomic heterogeneity between adult and pediatric $A L L$, including the different in the counts of gene mutation, the frequently mutated genes and the fusion genes, which may be the contributing factors that influence prognosis. This genomic landscape enhanced the understanding of the biological differences of disease between the two populations and provided a clue for novel therapeutic approaches.

\section{Declarations}

\section{Authors' contributions}

Yanxin Chen,Yongzhi Zheng and Yunda Hong collected the data. Jingjing Wen, Yunda Hong and Jiazheng Li analyzed the data. Yanxin Chen, Yan Huang and Yi Chen performed the experiments, Yanxin Chen, Yongzhi Zheng and Jianda Hu wrote the paper.

Data availability statement: The datasets generated during and/or analysed during the current study are available from the corresponding author on reasonable request.

Funding statement: This work was supported by National Natural Science Foundation of China (81870135, U2005204, 82000142), Natural Science Foundation of Fujian Province (2020J05049). Special Fund from Fujian Provincial Department of Finance (2016B041), Joint Funds for the Innovation of Science and Technology, Fujian province (2016Y9032).

Conflict of interest disclosure: The authors declare no conflict of interest.

Ethics approval statement: The study was approved by the Fujian Union Hospital Institutional Review Board. Informed consent or assent was obtained from all subjects and/or their legal guardians.

Patient consent statement: NA.

Permission to reproduce material from other sources: NA.

clinical trial registration: NA.

\section{References}


1. Zhang X, Li J, Lu P. Advances in the development of chimeric antigen receptor-T-cell therapy in B-cell acute lymphoblastic leukemia. Chinese medical journal 2020;133(4):474-82 doi $10.1097 / \mathrm{cm} 9.0000000000000638$.

2. Malard F, Mohty M. Acute lymphoblastic leukaemia. Lancet (London, England) 2020;395(10230):1146-62 doi 10.1016/s0140-6736(19)33018-1.

3. Bueno C, Montes R, Melen GJ, Ramos-Mejia V, Real PJ, Ayllon V, et al. A human ESC model for MLLAF4 leukemic fusion gene reveals an impaired early hematopoietic-endothelial specification. Cell Res 2012;22(6):986-1002 doi 10.1038/cr.2012.4.

4. Dieck CL, Tzoneva G, Forouhar F, Carpenter Z, Ambesi-Impiombato A, Sanchez-Martin M, et al. Structure and Mechanisms of NT5C2 Mutations Driving Thiopurine Resistance in Relapsed Lymphoblastic Leukemia. Cancer Cell 2018;34(1):136-47 e6 doi 10.1016/j.ccell.2018.06.003.

5. Gu Z, Churchman ML, Roberts KG, Moore I, Zhou X, Nakitandwe J, et al. PAX5-driven subtypes of Bprogenitor acute lymphoblastic leukemia. Nat Genet 2019;51(2):296-307 doi 10.1038/s41588-0180315-5.

6. Leon TE, Rapoz-D'Silva T, Bertoli C, Rahman S, Magnussen M, Philip B, et al. EZH2-Deficient T-cell Acute Lymphoblastic Leukemia Is Sensitized to CHK1 Inhibition through Enhanced Replication Stress. Cancer Discov 2020;10(7):998-1017 doi 10.1158/2159-8290.CD-19-0789.

7. Mullighan CG. New strategies in acute lymphoblastic leukemia: translating advances in genomics into clinical practice. Clin Cancer Res 2011;17(3):396-400 doi 10.1158/1078-0432.CCR-10-1203.

8. Braun TP, Eide CA, Druker BJ. Response and Resistance to BCR-ABL1-Targeted Therapies. Cancer Cell 2020;37(4):530-42 doi 10.1016/j.ccell.2020.03.006.

9. Pui $\mathrm{CH}$, Nichols KE, Yang JJ. Somatic and germline genomics in paediatric acute lymphoblastic leukaemia. Nat Rev Clin Oncol 2019;16(4):227-40 doi 10.1038/s41571-018-0136-6.

10. Paulsson K, Lilljebjorn H, Biloglav A, Olsson L, Rissler M, Castor A, et al. The genomic landscape of high hyperdiploid childhood acute lymphoblastic leukemia. Nat Genet 2015;47(6):672-6 doi 10.1038/ng.3301.

11. Yasuda T, Tsuzuki S, Kawazu M, Hayakawa F, Kojima S, Ueno T, et al. Recurrent DUX4 fusions in B cell acute lymphoblastic leukemia of adolescents and young adults. Nat Genet 2016;48(5):569-74 doi 10.1038/ng.3535.

12. Roberts KG, Gu Z, Payne-Turner D, McCastlain K, Harvey RC, Chen IM, et al. High Frequency and Poor Outcome of Philadelphia Chromosome-Like Acute Lymphoblastic Leukemia in Adults. J Clin Oncol 2017;35(4):394-401 doi 10.1200/JC0.2016.69.0073.

13. Andersson AK, Ma J, Wang J, Chen X, Gedman AL, Dang J, et al. The landscape of somatic mutations in infant MLL-rearranged acute lymphoblastic leukemias. Nat Genet 2015;47(4):330-7 doi 10.1038/ng.3230.

14. Brown PA, Shah B, Fathi A, Wieduwilt M, Advani A, Aoun P, et al. NCCN Guidelines Insights: Acute Lymphoblastic Leukemia, Version 1.2017. J Natl Compr Canc Netw 2017;15(9):1091-102 doi 10.6004/jnccn.2017.0147. 
15. Roberts KG. Genetics and prognosis of ALL in children vs adults. Hematology Am Soc Hematol Educ Program 2018;2018(1):137-45 doi 10.1182/asheducation-2018.1.137.

16. Liu YF, Wang BY, Zhang WN, Huang JY, Li BS, Zhang M, et al. Genomic Profiling of Adult and Pediatric B-cell Acute Lymphoblastic Leukemia. EBioMedicine 2016;8:173-83 doi 10.1016/j.ebiom.2016.04.038.

17. Coccaro N, Anelli L, Zagaria A, Specchia G, Albano F. Next-Generation Sequencing in Acute Lymphoblastic Leukemia. International journal of molecular sciences 2019;20(12) doi 10.3390/ijms20122929.

18. Tran TH, Hunger SP. The genomic landscape of pediatric acute lymphoblastic leukemia and precision medicine opportunities. Semin Cancer Biol 2020 doi 10.1016/j.semcancer.2020.10.013.

19. Nielsen R, Paul JS, Albrechtsen A, Song YS. Genotype and SNP calling from next-generation sequencing data. Nat Rev Genet 2011;12(6):443-51 doi 10.1038/nrg2986.

20. Liu Y, Easton J, Shao Y, Maciaszek J, Wang Z, Wilkinson MR, et al. The genomic landscape of pediatric and young adult T-lineage acute lymphoblastic leukemia. Nat Genet 2017;49(8):1211-8 doi 10.1038/ng.3909.

21. Mullighan CG. Genome sequencing of lymphoid malignancies. Blood 2013;122(24):3899-907 doi 10.1182/blood-2013-08-460311.

22. Molenaar R, Radivoyevitch T, Nagata Y, Khurshed M, Przychodzen B, Makishima H, et al. IDH1/2 Mutations Sensitize Acute Myeloid Leukemia to PARP Inhibition and This Is Reversed by IDH1/2Mutant Inhibitors. Clinical cancer research: an official journal of the American Association for Cancer Research 2018;24(7):1705-15 doi 10.1158/1078-0432.Ccr-17-2796.

23. Lemonnier F, Cairns R, Inoue S, Li W, Dupuy A, Broutin S, et al. The IDH2 R172K mutation associated with angioimmunoblastic T-cell lymphoma produces $2 \mathrm{HG}$ in $\mathrm{T}$ cells and impacts lymphoid development. Proceedings of the National Academy of Sciences of the United States of America 2016;113(52):15084-9 doi 10.1073/pnas.1617929114.

24. Hao Z, Cairns R, Inoue S, Li W, Sheng Y, Lemonnier F, et al. Idh1 mutations contribute to the development of T-cell malignancies in genetically engineered mice. Proceedings of the National Academy of Sciences of the United States of America 2016;113(5):1387-92 doi 10.1073/pnas. 1525354113.

25. Metzeler K, Herold T, Rothenberg-Thurley M, Amler S, Sauerland M, Görlich D, et al. Spectrum and prognostic relevance of driver gene mutations in acute myeloid leukemia. Blood 2016;128(5):686-98 doi 10.1182/blood-2016-01-693879.

26. Choi J, Goh G, Walradt T, Hong B, Bunick C, Chen K, et al. Genomic landscape of cutaneous T cell lymphoma. Nature genetics 2015;47(9):1011-9 doi 10.1038/ng.3356.

27. Bond J, Touzart A, Leprêtre S, Graux C, Bargetzi M, Lhermitte L, et al. DNMT3A mutation is associated with increased age and adverse outcome in adult T-cell acute lymphoblastic leukemia. Haematologica 2019;104(8):1617-25 doi 10.3324/haematol.2018.197848. 
28. Sanchez-Martin M, Ferrando A. The NOTCH1-MYC highway toward T-cell acute lymphoblastic leukemia. Blood 2017;129(9):1124-33 doi 10.1182/blood-2016-09-692582.

29. Caru M, Petrykey K, Drouin S, Beaulieu P, St-Onge P, Lemay V, et al. Identification of genetic association between cardiorespiratory fitness and the trainability genes in childhood acute lymphoblastic leukemia survivors. BMC cancer 2019;19(1):443 doi 10.1186/s12885-019-5651-z.

30. Ney GM, McKay L, Koschmann C, Mody R, Li Q. The Emerging Role of Ras Pathway Signaling in Pediatric Cancer. Cancer Res 2020;80(23):5155-63 doi 10.1158/0008-5472.CAN-20-0916.

31. Loftus J, Yahiaoui A, Brown P, Niswander L, Bagashev A, Wang M, et al. Combinatorial efficacy of entospletinib and chemotherapy in patient-derived xenograft models of infant acute lymphoblastic leukemia. Haematologica 2020 doi 10.3324/haematol.2019.241729.

32. Zhang W, Berthelet J, Michail C, Bui L, Gou P, Liu R, et al. Human CREBBP acetyltransferase is impaired by etoposide quinone, an oxidative and leukemogenic metabolite of the anticancer drug etoposide through modification of redox-sensitive zinc-finger cysteine residues. Free radical biology \& medicine 2020;162:27-37 doi 10.1016/j.freeradbiomed.2020.11.027.

33. Antić Ž, Yu J, Van Reijmersdal S, Van Dijk A, Dekker L, Segerink W, et al. Multiclonal complexity of pediatric acute lymphoblastic leukemia and the prognostic relevance of subclonal mutations. Haematologica 2020:0 doi 10.3324/haematol.2020.259226.

34. Elder A, Bomken S, Wilson I, Blair H, Cockell S, Ponthan F, et al. Abundant and equipotent founder cells establish and maintain acute lymphoblastic leukaemia. Leukemia 2017;31(12):2577-86 doi 10.1038/leu.2017.140.

\section{Tables}

Due to technical limitations, table 1 is only available as a download in the Supplemental Files section.

Table 2. The driver genes list of adult-ALL and pediatric-ALL 


\begin{tabular}{|c|c|}
\hline & Gene symbol \\
\hline $\begin{array}{l}\text { Adult- } \\
\text { ALL }\end{array}$ & 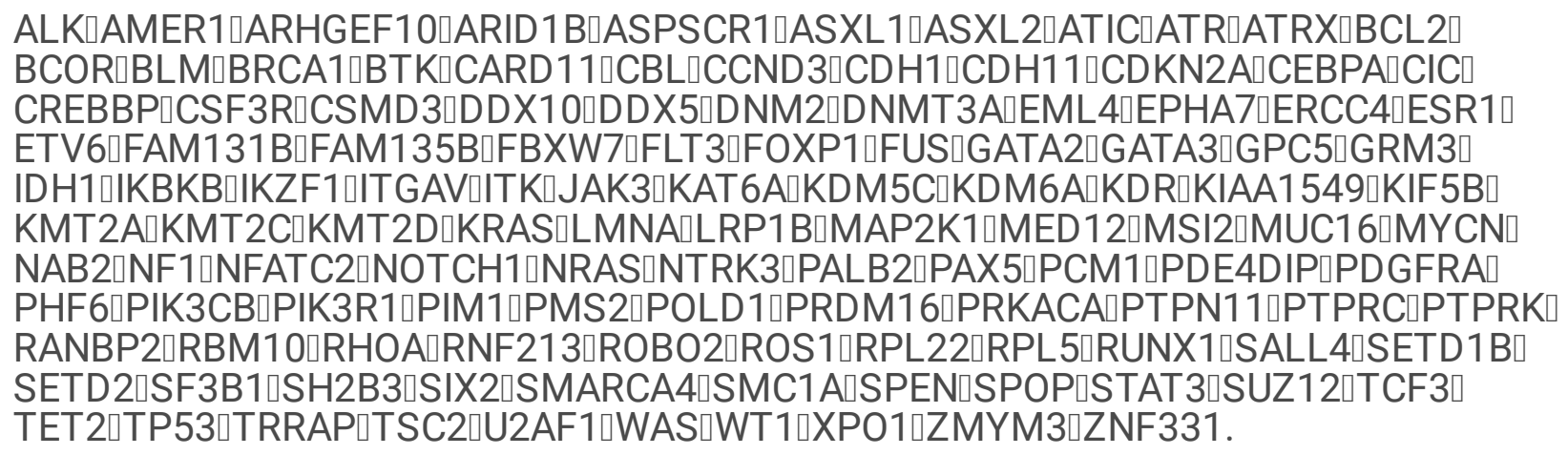 \\
\hline \multirow{6}{*}{$\begin{array}{l}\text { Pediatric- } \\
\text { ALL }\end{array}$} & ARID1B,ATRX,BCL11A,BCL11B,BCL3,BCL6,BRCA1,CACNA1D,CDH11, \\
\hline & CDKN1B,CDKN2A,CREBBP,CSF3R,CTCF,DEK,DICER1,DROSHA, \\
\hline & EP300,EZH2,FAS,FAT1,FBXW7,FLT3,FOX01,IDH1,IKZF1,JAK2,KDM5C, \\
\hline & KDM6A,LIFR,MAP3K1,MECOM,MLLT10,MYC,MYH9,NF1,NOTCH1, \\
\hline & NUMA1,PDGFB,PHOX2B,PLCG1,PML,POLE,PTPN11,RNF213,RNF43, \\
\hline & RUNX1,SF3B1,SMARCA4,STAT5B,WAS,XPC,ZNF521, \\
\hline
\end{tabular}

\section{Figures}

Figure 1

A

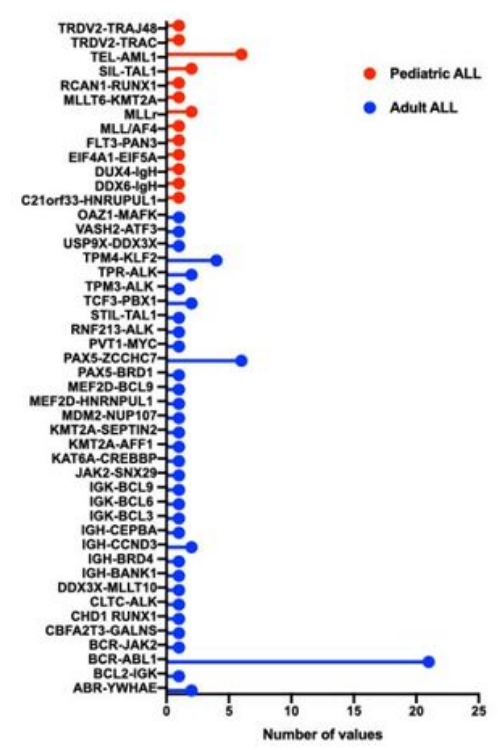

B

Adult ALL

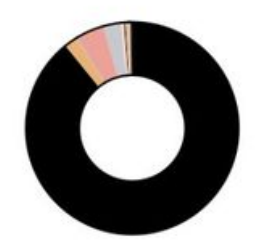

D

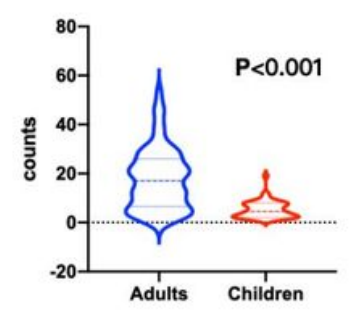

C

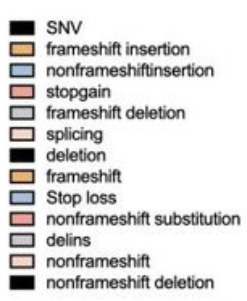

E

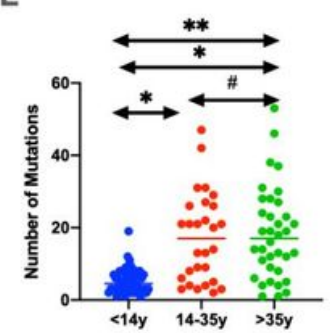

Pediatric ALL

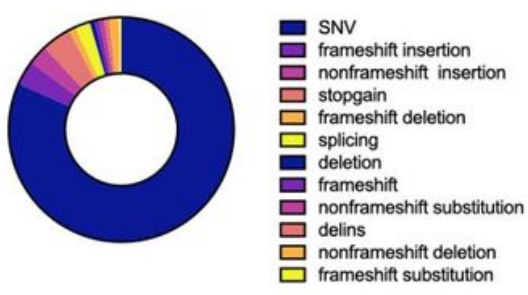

F

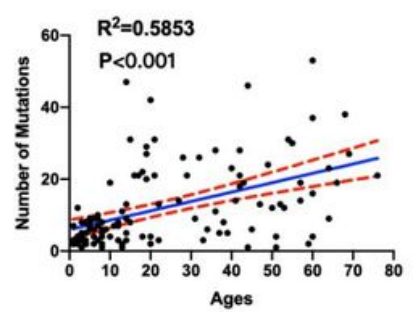

\section{Figure 1}


Comparison of gene rearrangements and mutation frequencies between adult and pediatric cases. (A)The Analysis of transcriptome sequencing data identified transcripts abnormal derived from 66 gene rearrangements in 38 (59.3\%) adult ALL samples and 20 gene rearrangements in 15 (27.7\%) pediatric ALL samples. The most frequent gene rearrangements were BCR/ABL1 fusions (18 samples), fusions of PAX5/ZCCH7 (6 samples) in adult ALL. The genomic mutation type in adult(B) and pediatric(C) ALL.

(D)Violin plot of mutation counts in adult and children $(P<0.001)$. The median number of detected gene mutations was 17 (range: 1-53) per sample in adult ALL and 4.5 (range: 1-19) in pediatric ALL. (E) Differences in the frequency of gene mutations between children $(<14 \mathrm{y})$, adolescent and young adult (14$35 \mathrm{y})$ and adult $(>35 \mathrm{y}) .\left({ }^{*}, P<0.05\right)$. (E)Correlation between mutation counts and age of ALL patients. A significant correlation between the number of gene mutations and age was found $(R 2=0.5853, P<0.001)$.

Figure 2

$\begin{array}{lll}\text { A } & \text { Adult ALL } & \text { Pediatric ALL }\end{array}$
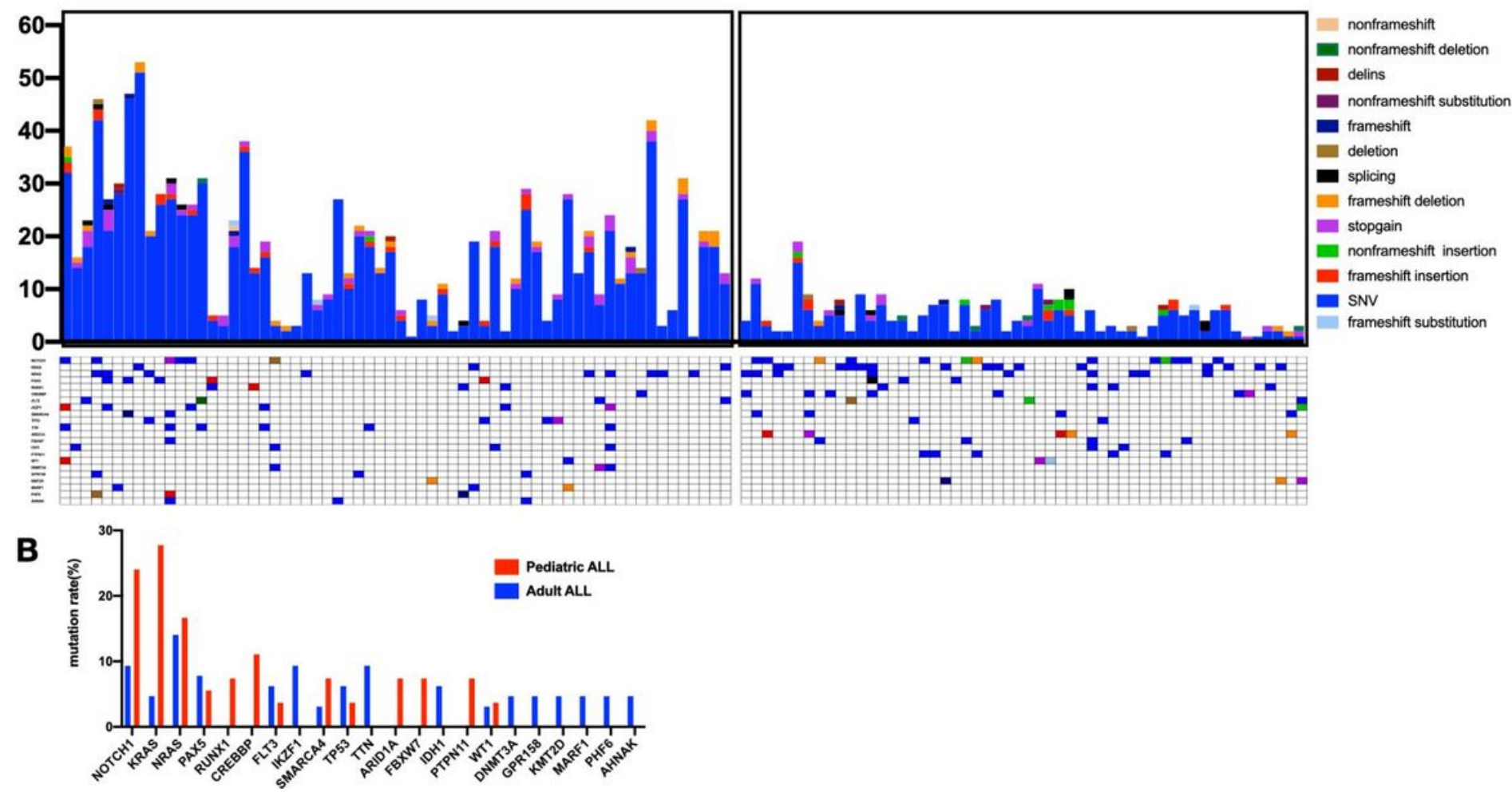

Figure 2

Comparison of genetic features between adult and pediatric cases. (A)The genomic landscape of adult and pediatric ALL. The mutational heatmap indicated the most recurrently altered genes in the $118 \mathrm{ALL}$ samples. Color coded by the genetic alteration types. (B) The percent of mutated genes in adult and pediatric ALL. The mutation rate was different in the adult and pediatric ALL. 
A

BP of driver genes in adult ALL

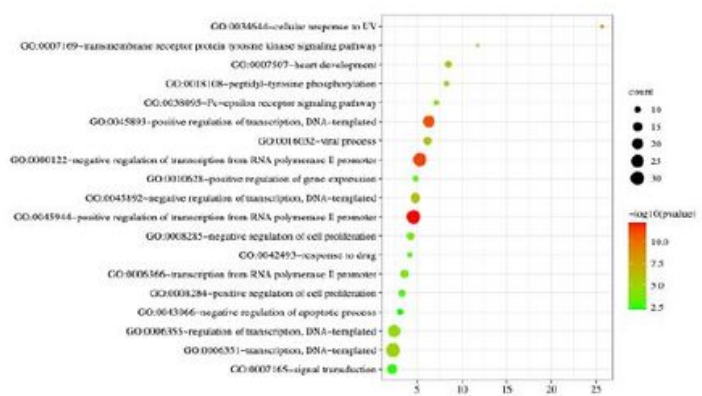

C

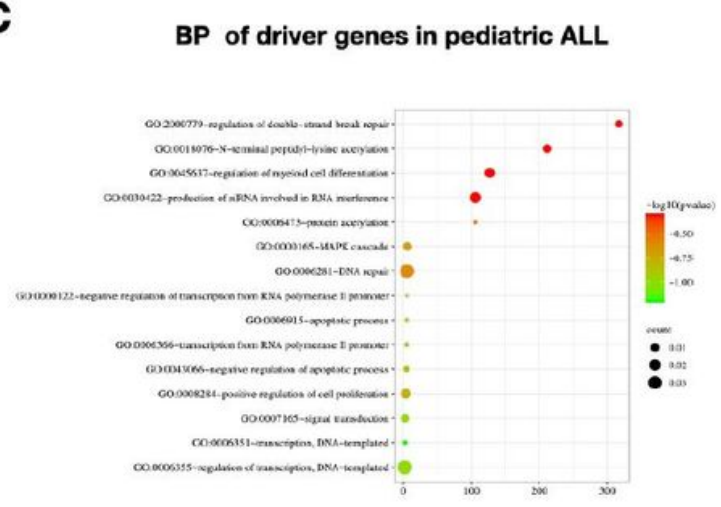

B

KEGG pathway of driver genes in adult ALL

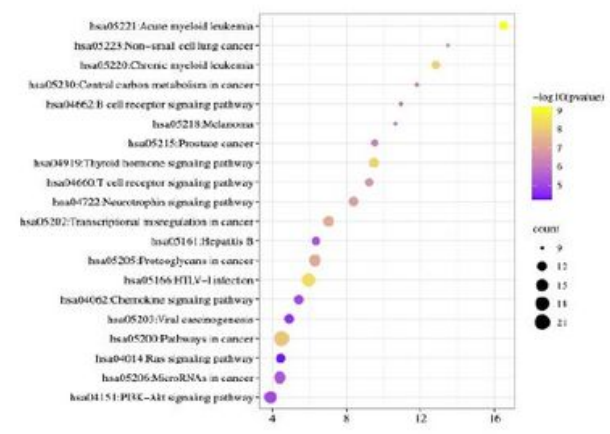

D

KEGG pathway of driver genes in pediatric ALL

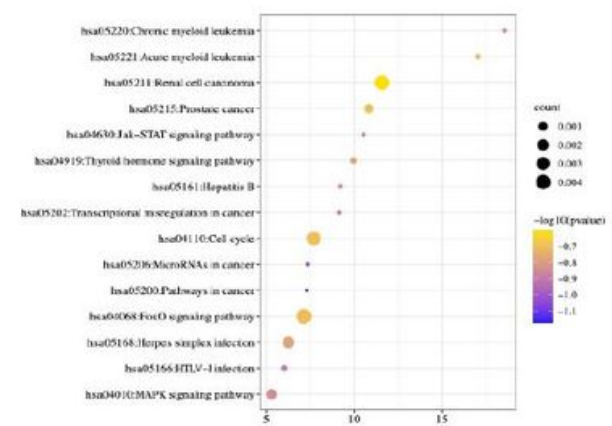

\section{Figure 3}

GO analysis and KEGG analysis of the adult and pediatric ALL. (A) Bubble diagram of significantly enriched GO terms of the biological process based on their functions in the adult ALL. (B) Bubble diagram of significantly enriched KEGG pathways in the adult ALL. (C) Bubble diagram of significantly enriched GO terms of the biological process in the pediatric ALL. (D) Bubble diagram of significantly enriched KEGG pathways in the pediatric ALL. 
A

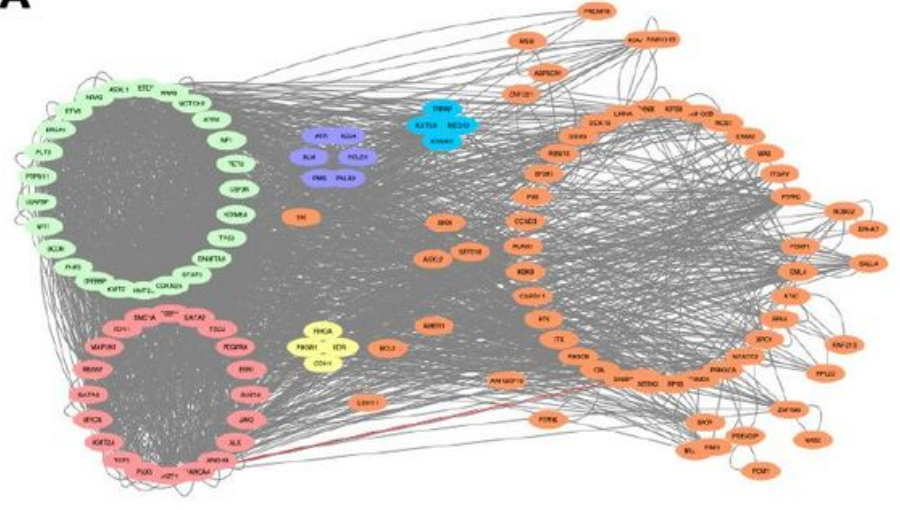

C

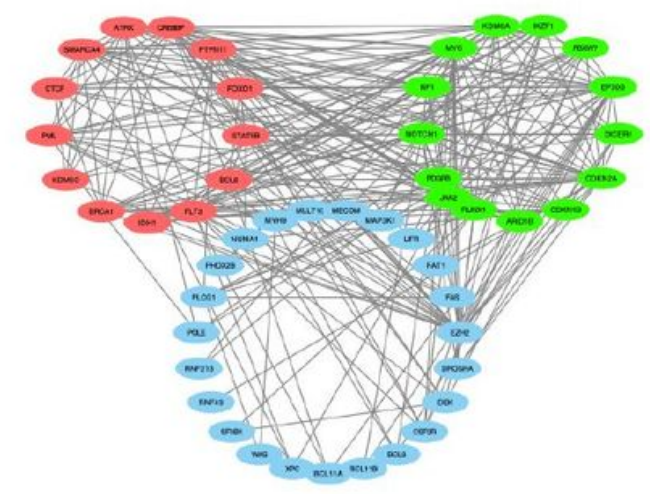

B

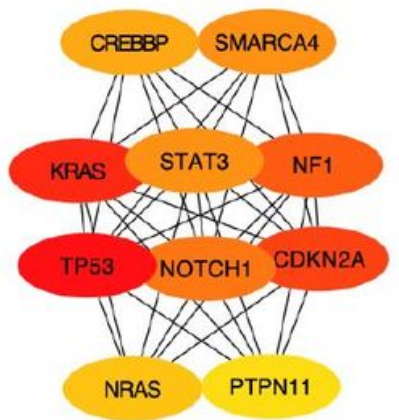

D

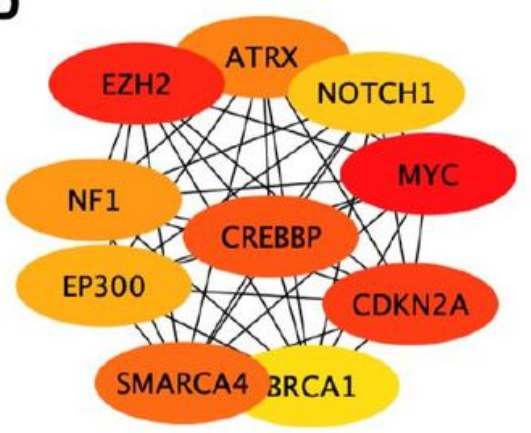

\section{Figure 4}

Driver genes PPI network complex based on String website and integrated by Cytoscape software. In this picture, each circle represents a gene (node) and each connection represents a direct or indirect connection (edge). (A) PPI network with MCODE analysis of the driver genes of adult ALL, clusters were extracted by MCODE and presented with different colors. green color represents cluster 1, pink color represents cluster 2, purple color represents cluster 3, yellow color represents cluster 4 , blue color represents cluster 4, and orange color represents un-clustered genes. (B) The top 10 of genes with highest degrees identified by cytoHubba analysis in the adult ALL cases. (C) PPI network i with MCODE analysis of the driver genes of pediatric ALL, clusters were extracted by MCODE and presented with different colors. Red color represents cluster 1 , green color represents cluster 2 , and blue color represents unclustered genes. (D) The top 10 of genes with highest degrees identified by cytoHubba analysis in the pediatric ALL cases. 
Figure 5

A

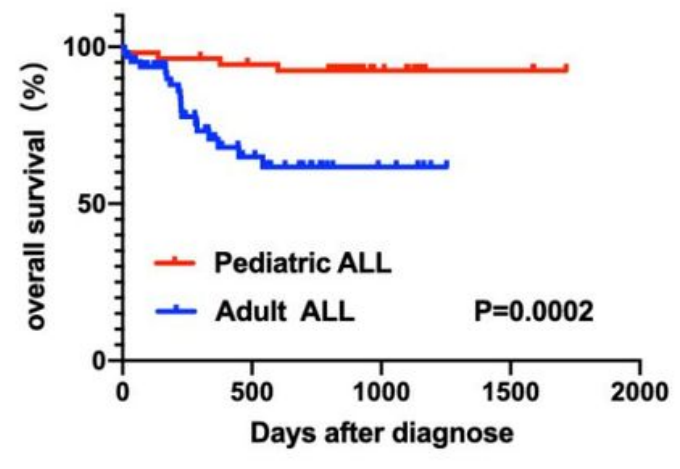

B

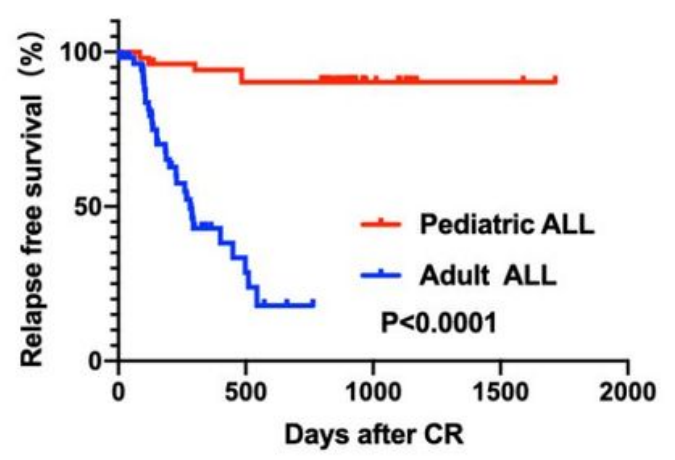

\section{Figure 5}

Associations of genetic features with clinical outcomes in adult and pediatric cases. (A) The OS of adult ALL were poorer than pediatric ALL $(P=0.0002)$.(B) The RFS of adult ALL were poorer than pediatric ALL $(P<0.001)(C)$ Correlation between mutation counts and times to relapse of ALL patients. 
Figure 6

A

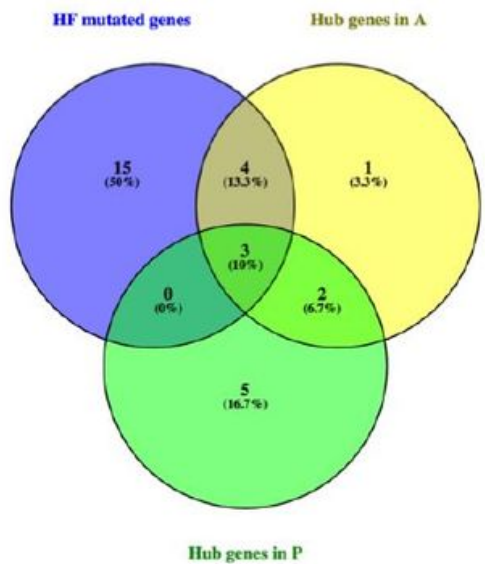

C

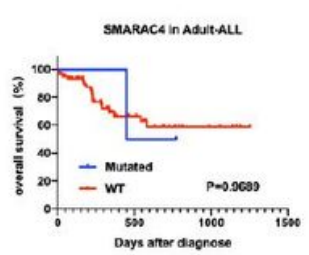

SMARCA in Peciatrie-ALL

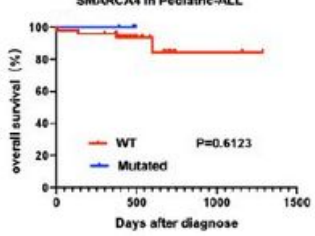

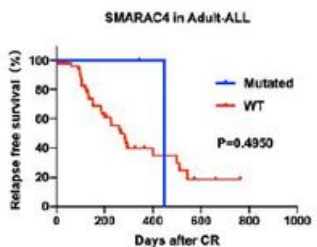

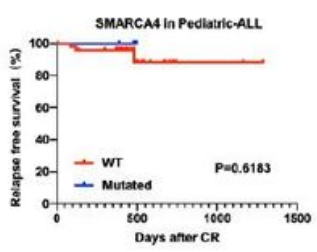

B
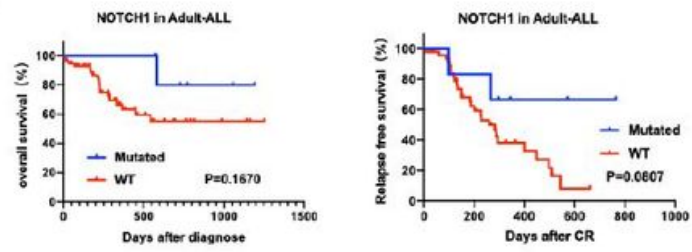

NOTCH1 in Podiatric-All
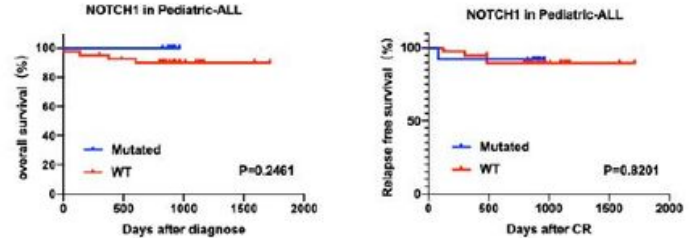

D

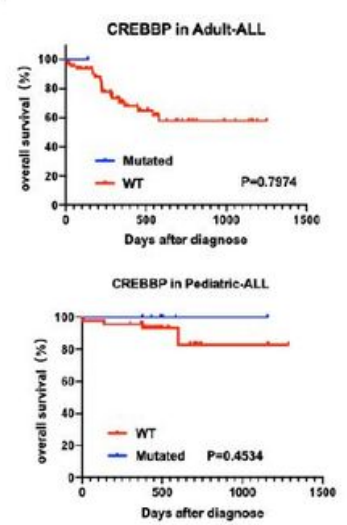

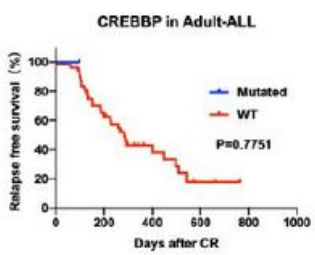

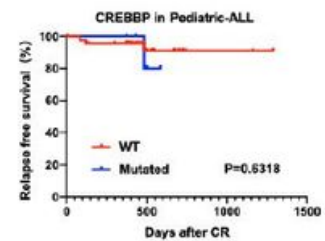

Figure 6

The survival analysis of the hub driver genes in adult ALL and pediatric ALL. (A) Vene plot of the overlapping genes between high frequency (HF) mutated genes and the hub driver genes of adult ALL and pediatric ALL. (B) The OS and RFS curve of the NOTCH1, CREBBP, and SMARCA4.

\section{Supplementary Files}

This is a list of supplementary files associated with this preprint. Click to download.

- Table1.jpg

- SupplementTable1.docx 